\title{
Mice, Models, Microbiota: How Can We More Accurately Reflect Human Disease?
}

\section{Rahman A. Ladak ${ }^{1, *}$ (D) and Dana Philpott ${ }^{1}$ (D)}
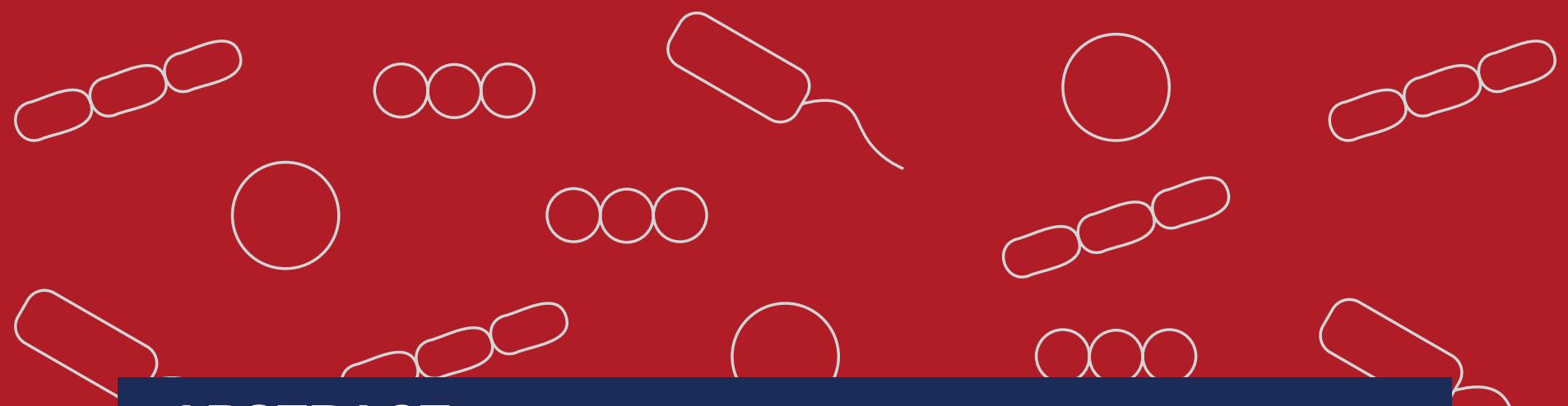

\section{ABSTRACT}

With growing evidence that human disease is affected by the microbiota, many researchers have sought to modulate the microbiomes of mice to improve translational research. Altering their microbiomes, which are usually germ-free or specific pathogen-free, might allow mice to more accurately model human disease and hence produce more applicable findings. However, this has been difficult to apply to individual projects due to the disparity of explained methods and results. In this review, we first describe the immunological functions of the gut microbiota and the methods of altering mice microbiota, from transplantation route to age of transplantation to microbiota source. We then present an approach for how the gut microbiota might be considered when modelling human disease in mice. By organizing findings by type of disease - neurological, immunological, chronic inflammatory, and cancer - we propose that mouse models can be improved by considering the source of the microbiota, the presence or absence of certain microbial phyla, and by timing the transplantation during a physiologically relevant stage of development, such as the first five weeks of life.

Keywords: Microbiota, Translational Research, Mouse Models, Disease Models, Microbiota Transplantation

Affiliations

${ }^{1}$ Department of Immunology, University of Toronto, Canada
${ }^{*}$ Please direct correspondence to:

Rahman A. Ladak

Email: Rahman.Ladak@mail.utoronto.ca

Published online on July 23, 2021. 


\section{WHY USE MOUSE MODELS? HISTORY OF TRANSLATIONAL RESEARCH}

Due to its small size, rapid breeding, and genetic tractability, the laboratory mouse (a genetic hybrid between Mus musculus domesticus and M. musculus musculus) is the animal model of choice for research on human disease. Their utility is conferred by their genetic similarity to humans, since they share $97.5 \%$ of their coding genome with us, and hence scientists assume that their metabolic and disease phenotypes likely resemble ours. Therefore, studies on disease phenotype and drug effectiveness in mouse models are predicted to 'translate' to humans.

\section{Translational Research in Mice}

When discrepancies between mouse models and their human counterparts were manifest in early studies, scientists were quick to blame genetics ${ }^{1}$. Comparing the different species' protein sequences to their functions, the mere $2.5 \%$ difference in the coding sequence were explained to alter the activities of proteins and their affinities to binding targets. Scientists therefore proposed that 'humanizing' key mouse cells could increase translational research value. 'Humanization' involves replacing mouse immune cells with human immune progenitor cells. An example is the PBMC humanized mouse, which expresses human peripheral mononuclear blood cells (PBMCs) instead of mouse ones, and therefore can be used as an in vivo model to study the human immune system².

This was hoped to solve the problem of low research translatability for mice in diseases affecting nonconserved pathways. However, 'humanization' of the mouse immune system was not a panacea to the problems of translational research. For example, mouse strains bred to model human disease as accurately as possible, such as the non-obese diabetic (NOD) mouse strain to model autoimmune diabetes, still have many differences between the mouse and human phenotypes. Since 'humanization' of mouse cells has failed to solve this problem, scientific consensus began to point towards other players in phenotype, the most prominent of which was the microbiome.

Typically, laboratory mice are housed under specificpathogen free (SPF) or germ-free (GF) conditions to prevent unpredictable disease outbreaks and to control for the effects of a potential pathogen on disease outcomes. Due to a complete absence of microbes, GF mice develop abnormalities of their immune systems ${ }^{3,4}$. While this problem exists to a lesser degree for SPF mice, they too show immune defects relative to their counterparts with wild-derived microbiotas as they often lack certain health-benefitting microbial species, such as segmented filamentous bacteria (SFBs) ${ }^{5}$.
Fortunately, the practice of ignoring the microbiota is now reversing; scientists are now focusing on adding instead of removing the microbiota to increase translational research value.

\section{TRANSPLANTATION METHODOLOGIES}

There are many procedures to perform microbiota transplantation, with varying effectiveness. The main procedure is oral-fecal transplantation, which involves orally gavaging (force-feeding) mice with fecal pellets. Though GF mice are usually used, mice with pre-existing microbiotas can be fed fecal pellets after repeated antibiotic treatments to displace the original microbiota of the mice. In general, oral antibiotics eliminate $90 \%$ of the pre-existing microbiome allowing the newly transplanted microbiota to engraft after a single oral transplant, though multiple fecal-oral transplants allow for greater stability in the new microbiota ${ }^{6,7}$.

Besides the method of transplantation, there are other variables that must be considered when transplanting microbiomes. They are summarized below:

The overall microbiome diversity. Microbiome alphadiversity, which indicates the number of different taxa within the community, dictates the diversity of antigens that can be provided to the immune system and can help mature the immune system into an adult-like form ${ }^{5}$. Ideally all mouse model research should be performed using microbiomes that are more diverse than GF or SPF mice.

The presence of certain key species. Each microbial species synthesizes its own metabolites, which can contribute to the training of the immune system by acting as antigens or by participating in modulating the immune response. There are a few key species that we know so far that impact immune development listed in Table 1.

The life stage of the mouse when transplantation occurs. Since irrecoverable development of the adaptive immune system occurs early in the fetal and postnatal period, transplanting a microbiota in the postnatal period (0-5 weeks) is critical for giving mice a healthy adaptive immune system. Nevertheless, transplantations during adulthood still achieve some changes to immune phenotype, especially regarding the innate immune system ${ }^{8}$.

\section{SOURCES OF MICROBIOTA USED IN TRANSPLANTATION}

The main sources for microbiota transplants are humans and 'dirty' mice, both of which have higher microbial diversity than typical lab mice. The term 'dirty' refers to more diverse microbiomes derived from wild 


\begin{tabular}{|l|l|l|l|}
\hline $\begin{array}{l}\text { Bacterial Species } \\
\text { or Taxa }\end{array}$ & $\begin{array}{l}\text { Immunomodulatory } \\
\text { Effects in Mice }\end{array}$ & Mechanism & References \\
\hline $\begin{array}{l}\text { Segmented } \\
\text { filamentous bacteria } \\
\text { (SFB) }\end{array}$ & $\begin{array}{l}\text { increase Th17 } \\
\text { effector pool }\end{array}$ & $\begin{array}{l}\text { produces SAA (serum } \\
\text { amyloid antigen) drives } \\
\text { Th17 differentiation by IL- } \\
\text { 1B induction }\end{array}$ & [14] [15] [16] \\
\hline Bacteroides fragilis & $\begin{array}{l}\text { increase IL-10+ T- } \\
\text { cells, increase Th1 } \\
\text { effector cells, reduce } \\
\text { Tregs }\end{array}$ & $\begin{array}{l}\text { Polysaccharide antigen } \\
\text { (PSA) }\end{array}$ & [17] [18] \\
\hline $\begin{array}{l}\text { Lactobacillus (L. } \\
\text { acidophilus, } L . \\
\text { casei, L. salivarius, } \\
\text { and L. lactis) }\end{array}$ & $\begin{array}{l}\text { anti-inflammatory } \\
\text { (many adaptive and } \\
\text { innate immune cells } \\
\text { involved) }\end{array}$ & $\begin{array}{l}\text { SCFAs and cell-surface } \\
\text { carbohydrates which } \\
\text { signal via TLRs }\end{array}$ & [19] [20] \\
\hline $\begin{array}{l}\text { Bacteroides (B. } \\
\text { bifidum and B. } \\
\text { lactis) }\end{array}$ & $\begin{array}{l}\text { Immunomodulation } \\
\text { and metabolic } \\
\text { function }\end{array}$ & SCFAs & [21] \\
\hline $\begin{array}{l}\text { Clostridium (clusters } \\
\text { IV, XIVa and XVIII) }\end{array}$ & Increases Tregs & SCFAs & [22] \\
\hline
\end{tabular}

Table 1 Bacterial species and taxa which exert immunomodulatory effects. The list includes common bacteria which play important immunomodulation roles.

mice or pet-store mice, since these organisms have the potential to drive greater immunological experience than cleaner laboratory GF or SPF mice. To confirm the differences between dirty and clean lab mice, one study assessed the number of CD8+ effector memory T-cells and found that immunological exposure of the lab mice to the microbes from pet-stores during cohousing increased the population of these cells ${ }^{9}$. This indicates that the microbiota of the pet-store mice was outcompeting the lab microbiotas, but not the reverse, since the laboratory mouse microbiome failed to transplant into the wild mouse ${ }^{10}$.

Another factor to consider is the species-specific relationship of the microbiota being transplanted. Dirty microbiotas that are adapted specifically to Mus musculus mice can transplant successfully into typical lab mice, whereas human microbiomes cannot transplant as successfully due to inter-special differences in microbial composition and function ${ }^{11}$. Instead, the composition of the human microbiome when transplanted into mice changes into a new stable state, where phyla related to pre-existing mouse phyla enrich and microbes with metabolites or functions unique to humans are reduced or eliminated. For example, transplantation of asthmatic human microbiomes with genera Bacteroidaceae, Peptostreptococcaeae, and Clostridiaceae into mice that lacked those genera resulted in successful colonization of the first two, but no colonization of the third genera since no mousespecific Clostridiaceae spp. niche developed ${ }^{12}$. While this alteration in the microbiota to a mouse-like state may be in part due to the dietary differences from humans, it will nonetheless have consequences on host phenotype if important immunomodulatory phyla are lost or enriched ${ }^{13}$.

\section{AUTOIMMUNE CHRONIC DISEASE MODELS}

Transplanting the human microbiota into mice is another solution to increase research translatability. Due to the bidirectional relationship between disease and the gut microbiota, dysbiosis in the gut microbiome impacts the immune system. Therefore, transplanting dysbiotic microbiotas from humans into mice may replicate this immune phenotype in mice. This section will attempt to summarize how this can be executed successfully, and with what limitations.

\section{Multiple Sclerosis (MS)}

Multiple sclerosis (MS) is a neurodegenerative and inflammatory disease where immune cells, both innate and adaptive, destroy the myelin sheath of neurons in the central nervous system. One commonly used mouse model for multiple sclerosis, experimental autoimmune encephalitis (EAE), mimics the human disease process by activating CD4+ T helper cells with specificity towards myelin proteins. The EAE model mice are usually raised in specific pathogen-free (SPF) conditions since germ-free (GF) mice fail to develop $E A E^{14}$. Thus, even before conducting experiments with microbiota transplantation, it is evident that the microbiota affects EAE mice as its presence seems to 
be a prerequisite for disease.

Improving the EAE model by using human-transplanted microbiota relies on the presence of specific microbial taxa. MS microbiotas are enriched in $A$. municiphila and Acinetobacter genera and are reduced in Parabacteroides (i.e. P. distasonis) relative to MSfree counterparts. During microbiota transplants, $A$. municiphila did not colonize mice, but Acinetobacter calcoaceticus did. Their colonization reduced Treg cells and increased IFNy+ Th1 cells, hence indicating a shift from anti-to pro-inflammatory state. Likewise, a separate procedure involving $P$. distasonis transplantation led to increased IL-10 levels, as observed in humans, confirming the IL-10 dependent mechanisms of gut microbes in causing MS pathology. These results are instructive for microbiota-enhanced disease modelling at large since they show that the effects of an individual species in disease pathophysiology are key to mouse modelling. Importantly, in showing that not all humantransplanted bacteria can colonize mice, they also reiterate that mouse models can only model - and not replicate - human disease ${ }^{15}$.

\section{Inflammatory Bowel Disease (IBD)}

Inflammatory bowel disease results in destruction of the colon's absorptive abilities. Though genetic risk factors can influence whether someone develops the disease ${ }^{16}$, research has shown that microbial dysbiosis can also promote the development of IBD ${ }^{17}$. One study transplanted human microbiomes from IBD patients into model mice and observed an increase in Th17/Th2 cells and a decrease in Tregs, matching patterns observed in humans ${ }^{18}$. Similar experiments have identified other relationships between the microbiota and disease progression ${ }^{19,20,21}$, prompting researchers to investigate the role of the microbiome as a therapeutic platform. The current models for IBD research are usually in vitro cell cultures or laboratory mice, both of which lack the research translatability of humanized microbiota mice ${ }^{20}$.

Although some difficulties have arisen where human microbiota transplants into mice led to variable effects, such as microbiotas from IBD remission patients failing to confer remission in IBD in mice ${ }^{21}$, the general trend has been that transplanting human IBD microbiotas into mice exacerbates IBD-like symptoms ${ }^{23,24}$. Humanized microbiota mice are already being used to discover drugs, such as PACAP (Pituitary adenylate cyclaseactivating polypeptide), which has succeeded in preclinical mouse trials and is set to be used in human clinical trials ${ }^{25}$.

\section{CANCERS}

Although cancer is a genetic disease, its prognosis and treatment are impacted by the gut microbiota. This is because the microbiota impacts inflammation and immunity systemically and not only at the level of the intestine, and thus may play a role in carcinogenic inflammatory processes. An introductory review on the relationships of the microbiome to cancer development and therapy is given by Nishimura et al., 201926. This review will focus on the relationship of the microbiota to 3 of the most common cancers (colorectal, lung, and breast).

\section{Colorectal Cancer}

Although microbiome-host interactions occur for cancers around the body, they are most easily studied with respect to colorectal cancer (CRC) since the cancer is directly in contact with the intestinal microbiota. Certain microbiome profiles have been linked to the adenoma-adenocarcinoma sequence, which involves genomic instability triggered by the inactivation of tumor-suppressor genes, including APC (adenomatous polyposis coli), and further studies have identified the specific microbial species responsible for driving this mutational sequence ${ }^{27}$.

The relationship between $\mathrm{CRC}$ development in humans and the microbiota involves a few key bacterial species, namely Fusobacterium nucleatum, Bacteroides fragilis, and pathogenic colibactin-expressing E. coli among others ${ }^{28,29}$. While many of these species produce metabolites that alter the gene expression profiles of colorectal epithelial cells to help trigger cancer, the key driver of CRC mutation is likely F. nucleatum, which has an adhesin called FadA that binds to E-cadherin and increases $\beta$-catenin signaling to trigger epithelial cell growth $^{30}$. The effects of $F$. nucleatum are localized to CRC cells since they have a receptor, Fap2, for CRC's upregulated Gal-GalNAc glycoprotein ${ }^{31,32}$, thereby enriching F. nucleatum at sites of colorectal tumors.

\section{Lung Cancer}

Lung cancer has been linked to microbial dysbiosis. This includes the gut microbiome (gut-lung axis) as well as the local lung microbiota. Researchers determined that the lung microbiota plays a role in the acceleration of cancer development by inducing a pro-inflammatory environment. Jacks et al. (2018) gave antibiotics to mice with oncogenic mutations and found that antibiotic treatment reduced tumor growth ${ }^{33}$. The researchers also identified an increase in Herbaspirillum and Sphingomonadaceae bacteria in cancerous mouse lungs, which differs from the observed increase in Helicobacter pylori and F. nucleatum in human lung tumors. To increase correspondence between human and mouse lung microbiotas, a feasibility test was performed by transplanting human $\mathrm{H}$. pylori into healthy mouse lungs. This induced a cancer-associated 

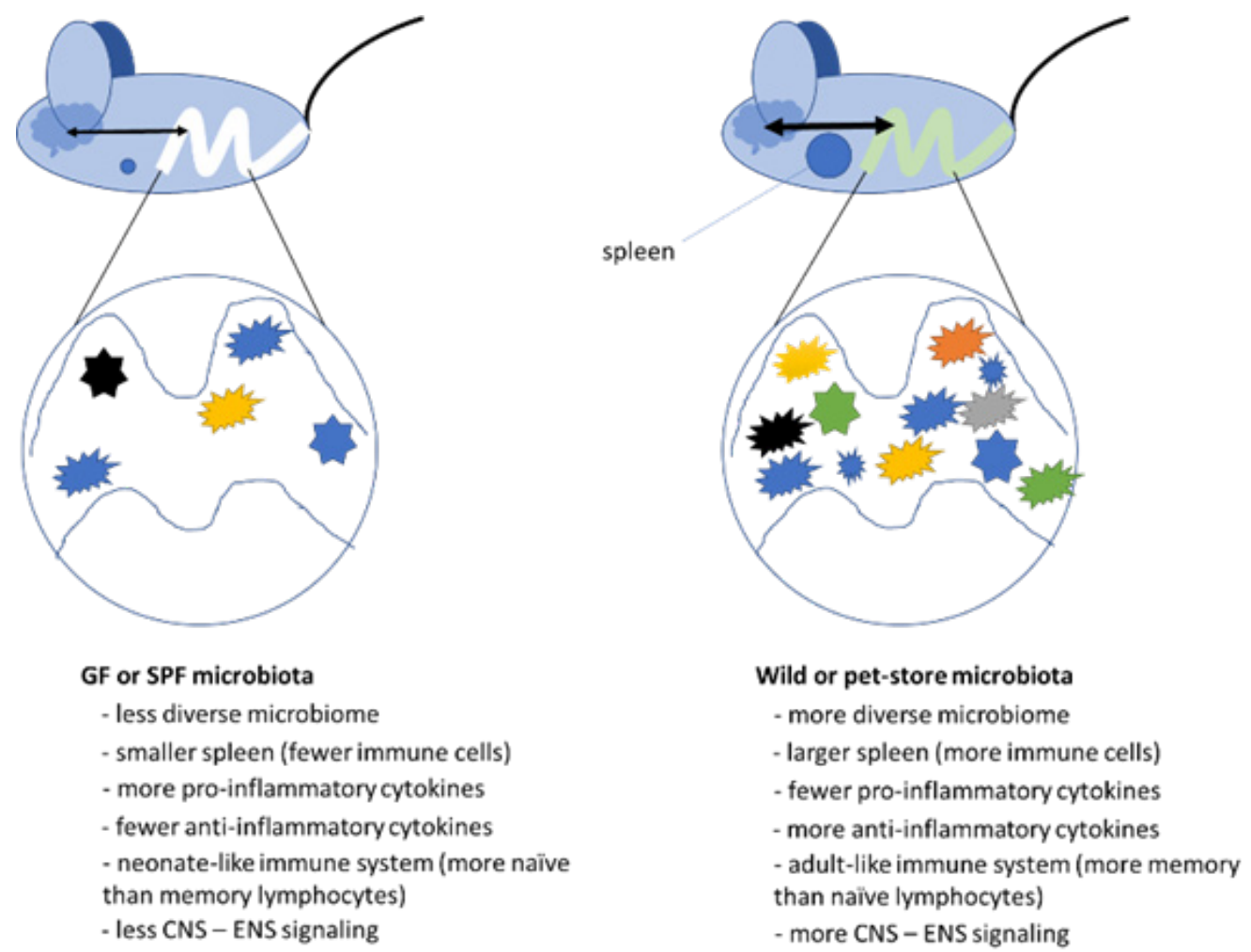

Figure 1 Mice with more diverse microbiomes (for example, mice colonized with a microbiota derived from a wild mouse) develop an adult-like immune system with more effector and memory $\mathrm{T}$ and $\mathrm{B}$ lymphocytes, and increased monocytes, granulocytes, and ILCs in peripheral lymphoid and non-lymphoid tissues. There are also fewer pro-inflammatory cytokines and more anti-inflammatory cytokines as the Treg population is increased. A more diverse microbiota leads to more autonomic nervous signaling between the brain and gut. Although this is not labelled in the diagram, a more diverse microbiota also impacts the endocrine system by producing more endocrine hormone analogues.

cytokine profile ${ }^{34}$, meaning that humanized lung microbiomes could potentially be used as a humanized microbiota lung cancer mouse model.

Interestingly, the above experiments focused only on the lung microbiome and concluded that microbiome effects in lung cancer are local, since the fecal bacterial load remained unaltered. This conclusion fails to recognize the significance of the gut microbiota in impacting the prognosis of lung cancer, since the intestinal microbiome has systemic effects on immunity and inflammation. Hence, intestinal probiotics should be tested for their ability to modulate cancer development. One example is the use of cisplatin, which does not significantly reduce tumors in mice unless administered with the human-derived bacteria L. acidophilus ${ }^{35}$, elucidating an improvement to an existing chemotherapy.

\section{Breast Cancer}

Breast cancer is the most common cancer in adult women and one major variable involved in breast cancer progression is the microbiota. For example, the bacterial species, Helicobacter hepaticus, increases neutrophil recruitment to the breast tissues leading to cancer development in certain mouse models ${ }^{36}$. Another mechanism involves production of estrogen-like compounds by a few human bacterial species, such as Escherichia coli, Klebsiella sp-1-1-55, Prevotella amnii, Enterococcus gallinarum, Actinomyces sp. HPA0247, Shewanella putrefaciens, and Erwinia amylovora ${ }^{37}$. These bacteria provide estrogen-like compounds by metabolizing dietary lignans into estrogen-like compounds such as enterodiol or enterolactone that alter the growth of breast tumors ${ }^{38,39}$.

\section{DRUG-RESPONSE MODELLING IN MICE}

Due to the importance of the microbiota in modulating the immune system and metabolizing drugs, preclinical trials of chemotherapeutics should be performed in humanized microbiota animals. Preclinical studies in conventional lab mice often fail to take the microbiota into account, which may contribute to the low rate of translation of drugs from mouse models to humans. 
It is plausible that some human-specific bacteria are metabolizing the drug in an unforeseen way.

In the case of synthetic monoclonal antibodies against CTLA-4 (a checkpoint inhibitor used as an anti-cancer chemotherapeutic agent), genetically similar mice from different facilities had microbiotas differing in relative abundance of Bifidobacterium spp. and increases in Bifidobacterium frequency were shown to cause increased efficacy of the drug. This finding matched a finding in humans, where the presence of the species $B$. thetaiotaomicron and $B$. fragilis conferred drug responsivity ${ }^{40}$. Similarly, with checkpoint inhibitors of the PD-1/PD-L1 axis, drug efficacy was shown to be related to the presence or absence of the $A$. muciniphila, since it increased IL12 and CD4+ T-cells, which are needed to achieve the full drug response. These findings stress that translatability of results depends on the resemblance of the microbiotas of mice to their human equivalents. If warranted, personalized medicine can also be performed using humanized microbiota mice for each patient, where microbiome profiles can be investigated to see how the drug will be metabolized ${ }^{41,42}$. A summary of microbial taxa associated with responsiveness or non-responsiveness to immune checkpoint inhibitors is given by Wu Kongming et al. (2018) ${ }^{43}$.

\section{LIMITATIONS AND FUTURE DIRECTIONS}

While studies with human-microbiota associated (HMA) mice may increase research translatability, immunological and physiological deficiencies in HMA mice remain that may confound experimental finding $\mathrm{s}^{44}$. These differences arise from the specificity of the host-microbiota relationship-only $15 \%$ of bacterial strains and $4 \%$ of microbiome genes are shared between both mice and humans ${ }^{41}$. Even when a given bacterial species is shared between two hosts, such as segmented filamentous bacteria (SFB) present in both mice and rats, interchanging the species-specific strains abrogates their functions; indeed, SFB from rat intestines fails to induce Th17 development in mice, highlighting the existence of strain differences among bacterial species ${ }^{5}$. Given that human microbiotas are even more different from mouse ones, non-specific host-microbe interactions in HMA mice are an obvious confounding variable.

To overcome these limitations with HMA mice, the following approaches should be considered. The inspiration for the following was taken from a review by Brett Finlay et al., (2016) ${ }^{47}$. Firstly, when a healthy state is being researched using mice, such as ecological studies about infections, diet, or genetics, it is recommended to use a wild-mouse microbiota. Because wild mice share the similar immunomodulatory bacteria as lab mice, yet have a higher diversity, transplanting their microbiome will lead to better immune training. Secondly, if a human disease is being modeled in mice, using a disease-specific microbiota transplant from humans, and transplanting during the neonatal window of opportunity will provide the most translatable mouse model. Next, to ensure functional similarity between the transplanted microbiota in the recipient and in the donor, the metabolic profiles of the pre-transplantation and post-transplantation microbiotas should be compared. Although the usual protocol is to assess the relative abundance of different phyla by OTUs (operational transcriptional units, from $16 \mathrm{~S}$ rDNA sequencing), this comparison is not as good as comparing the metabolic profiles since the microbial metabolites are what exert their mechanisms of action on the host and the metabolic profiles of structurally different microbiotas may be similar. Even if the OTU abundances differ between the donor and recipient, the microbiotas are functionally similar if their metabolic profiles are similar. Finally, humanized microbiota animals are not a replacement for existing animal models; instead, they should be viewed as an addition to the repertoire of diverse models, each with their own specific uses. As discussed with the MS-microbiota transplant mice, there are functional differences between the donor and recipient microbiotas and using multiple mouse models in studies may increase translational research value since different mouse models make up for one another's limitations.

\section{ACKNOWLEDGEMENTS}

I would like to thank Professor Dana J. Philpott from the Immunology department at the University of Toronto for acting as a supervisor for this project. This paper was completed as an extension to the two-year Trinity One seminar course series, coordinated by Trinity College at the University of Toronto. This current manuscript is a condensed version of the original paper, of which many primary citations came from Professor Philpott's research team. Her other contributions include helping develop the main topic of the paper, providing stylistic edits, and other personal guidance during the process of writing and publishing this manuscript.

\section{COMPETING INTERESTS}

No competing interests declared.

Received: January 29, 2021

Accepted: June 11, 2021

Published online: July 23, 2021

\section{REFERENCES}

1. Perlman, R. L. (2016). Mouse models of human disease: An evolutionary perspective. Evol Med Public Health, 2016(1), 170176. doi:10.1093/emph/eow014 
2. Li, Y., \& Di Santo, J. P. (2019). Modeling Infectious Diseases in Mice with a "Humanized" Immune System. Microbiol Spectr, 7(2). doi:10.1128/microbiolspec.BAI-0019-2019

3. Arrieta, M. C., Stiemsma, L. T., Amenyogbe, N., Brown, E. M., \& Finlay, B. (2014). The intestinal microbiome in early life: health and disease. Front Immunol, 5, 427. doi:10.3389/fimmu.2014.00427

4. Gensollen, T., Iyer, S. S., Kasper, D. L., \& Blumberg, R. S. (2016). How colonization by microbiota in early life shapes the immune system. Science, 352(6285), 539-544. doi:10.1126/science. aad9378

5. Tan, T. G., Sefik, E., Geva-Zatorsky, N., Kua, L., Naskar, D., Teng, F., . . . Mathis, D. (2016). Identifying species of symbiont bacteria from the human gut that, alone, can induce intestinal Th17 cells in mice. Proc Natl Acad Sci U S A, 113(50), E8141-E8150. doi:10.1073/pnas.1617460113

6. Staley, C., Kaiser, T., Beura, L. K., Hamilton, M. J., Weingarden, A. R., Bobr, A., . . Khoruts, A. (2017a). Stable engraftment of human microbiota into mice with a single oral gavage following antibiotic conditioning. Microbiome, 5(1), 87. doi:10.1186/s40168017-0306-2

7. Wrzosek, L., Ciocan, D., Borentain, P., Spatz, M., Puchois, V., Hugot, C., . . . Cassard, A. M. (2018). Transplantation of human microbiota into conventional mice durably reshapes the gut microbiota. Sci Rep, 8(1), 6854. doi:10.1038/s41598-018-25300-3 8. Tan, T. G., Sefik, E., Geva-Zatorsky, N., Kua, L., Naskar, D., Teng, F., . . . Mathis, D. (2016). Identifying species of symbiont bacteria from the human gut that, alone, can induce intestinal Th17 cells in mice. Proc Natl Acad Sci U S A, 113(50), E8141-E8150. doi:10.1073/pnas.1617460113

9. Robertson, S. J., Lemire, P., Maughan, H., Goethel, A., Turpin, W., Bedrani, L., . . . Philpott, D. J. (2019). Comparison of Cohousing and Littermate Methods for Microbiota Standardization in Mouse Models. Cell Rep, 27(6), 1910-1919.e1912. doi:10.1016/j. celrep.2019.04.023

10. Beura, L. K., Hamilton, S. E., Bi, K., Schenkel, J. M., Odumade, O. A., Casey, K. A., . . . Masopust, D. (2016). Normalizing the environment recapitulates adult human immune traits in laboratory mice. Nature, 532(7600), 512-516. doi:10.1038/nature17655

11. Rosshart, S. P., Herz, J., Vassallo, B. G., Hunter, A., Wall, M. K., Badger, J. H., . . . Rehermann, B. (2019b). Laboratory mice born to wild mice have natural microbiota and model human immune responses. Science, 365(6452). doi:10.1126/science.aaw4361

12. Arrieta, M. C., Stiemsma, L. T., Dimitriu, P. A., Thorson, L., Russell, S., Yurist-Doutsch, S., . . . Investigators, C. S. (2015a). Early infancy microbial and metabolic alterations affect risk of childhood asthma. Sci Transl Med, 7(307), 307ra152. doi:10.1126/ scitranslmed.aab2271

13. Carmody, R. N., Gerber, G. K., Luevano, J. M., Gatti, D. M., Somes, L., Svenson, K. L., \& Turnbaugh, P. J. (2015). Diet dominates host genotype in shaping the murine gut microbiota. Cell Host Microbe, 17(1), 72-84. doi:10.1016/j.chom.2014.11.010

14. Atarashi, K., Tanoue, T., Oshima, K., Suda, W., Nagano, Y., Nishikawa, H., . . . Honda, K. (2013a). Treg induction by a rationally selected mixture of Clostridia strains from the human microbiota. Nature, 500(7461), 232-236. doi:10.1038/nature12331

15. Colpitts, S. L., Kasper, E. J., Keever, A., Liljenberg, C., Kirby, T., Magori, K., . . . Ochoa-Repáraz, J. (2017). A bidirectional association between the gut microbiota and CNS disease in a biphasic murine model of multiple sclerosis. Gut Microbes, 8(6), 561-573. doi:10.1080/19490976.2017.1353843

16. Arrieta, M. C., Sadarangani, M., Brown, E. M., Russell, S. L., Nimmo, M., Dean, J., . . Finlay, B. B. (2016b). A humanized microbiota mouse model of ovalbumin-induced lung inflammation. Gut Microbes, 7(4), 342-352. doi:10.1080/19490976.2016.118229 3

17. Etienne-Mesmin, L., Chassaing, B., \& Gewirtz, A. T. (2017). Tryptophan: A gut microbiota-derived metabolites regulating inflammation. World J Gastrointest Pharmacol Ther, 8(1), 7-9. doi:10.4292/wjgpt.v8.i1.7

18. Shah, R., Cope, J. L., Nagy-Szakal, D., Dowd, S., Versalovic, J., Hollister, E. B., \& Kellermayer, R. (2016). Composition and function of the pediatric colonic mucosal microbiome in untreated patients with ulcerative colitis. Gut Microbes, 7(5), 384-396. doi:10 .1080/19490976.2016.1190073

19. Reinoso Webb, C., den Bakker, H., Koboziev, I., Jones-Hall, Y., Rao Kottapalli, K., Ostanin, D., . . . Grisham, M. B. (2018). Differential Susceptibility to T Cell-Induced Colitis in Mice: Role of the Intestinal Microbiota. Inflamm Bowel Dis, 24(2), 361-379. doi:10.1093/ibd/izx014

20. Roy, U., Gálvez, E. J. C., Iljazovic, A., Lesker, T. R., Błażejewski, A. J., Pils, M. C., . . . Strowig, T. (2017). Distinct Microbial Communities Trigger Colitis Development upon Intestinal Barrier Damage via Innate or Adaptive Immune Cells. Cell Rep, 21(4), 994-1008. doi:10.1016/j.celrep.2017.09.097

21. Geirnaert, A., Calatayud, M., Grootaert, C., Laukens, D., Devriese, S., Smagghe, G., . . Van de Wiele, T. (2017a). Butyrateproducing bacteria supplemented in vitro to Crohn's disease patient microbiota increased butyrate production and enhanced intestinal epithelial barrier integrity. Sci Rep, 7(1), 11450. doi:10.1038/ s41598-017-11734-8

22. Hall, A. B., Yassour, M., Sauk, J., Garner, A., Jiang, X., Arthur, T., . . . Huttenhower, C. (2017). A novel Ruminococcus gnavus clade enriched in inflammatory bowel disease patients. Genome Med, 9(1), 103. doi:10.1186/s13073-017-0490-5

23. Basson, A. R., Gomez-Nguyen, A., Menghini, P., Buttó, L. F., Di Martino, L., Aladyshkina, N., . . . Cominelli, F. (2020). Human Gut Microbiome Transplantation in lleitis Prone Mice: A Tool for the Functional Characterization of the Microbiota in Inflammatory Bowel Disease Patients. Inflamm Bowel Dis, 26(3), 347-359. doi:10.1093/ibd/izz242

24. Britton, G. J., Contijoch, E. J., Mogno, I., Vennaro, O. H., Llewellyn, S. R., Ng, R., . . . Faith, J. J. (2019). Microbiotas from Humans with Inflammatory Bowel Disease Alter the Balance of Gut Th17 and RORyt. Immunity, 50(1), 212-224.e214. doi:10.1016/j. immuni.2018.12.015

25. Li, J., Butcher, J., Mack, D., \& Stintzi, A. (2015). Functional impacts of the intestinal microbiome in the pathogenesis of inflammatory bowel disease. Inflamm Bowel Dis, 21(1), 139-153. doi:10.1097/MIB.0000000000000215

26. Nemetz, N., Abad, C., Lawson, G., Nobuta, H., Chhith, S., Duong, L., . . . Waschek, J. A. (2008). Induction of colitis and rapid development of colorectal tumors in mice deficient in the neuropeptide PACAP. Int J Cancer, 122(8), 1803-1809. doi:10.1002/ ijc. 23308

27. Nagano, T., Otoshi, T., Hazama, D., Kiriu, T., Umezawa, K., Katsurada, N., \& Nishimura, Y. (2019). Novel cancer therapy 
targeting microbiome. Onco Targets Ther, 12, 3619-3624. doi:10.2147/OTT.S207546

28. Tilg, H., Adolph, T. E., Gerner, R. R., \& Moschen, A. R. (2018). The Intestinal Microbiota in Colorectal Cancer. Cancer Cell, 33(6), 954-964. doi:10.1016/j.ccell.2018.03.004

29. Kostic, A. D., Chun, E., Robertson, L., Glickman, J. N., Gallini, C. A., Michaud, M., . . . Garrett, W. S. (2013a). Fusobacterium nucleatum potentiates intestinal tumorigenesis and modulates the tumor-immune microenvironment. Cell Host Microbe, 14(2), 207215. doi:10.1016/j.chom.2013.07.007

30. Gagnière, J., Raisch, J., Veziant, J., Barnich, N., Bonnet, R., Buc, E., . . Bonnet, M. (2016). Gut microbiota imbalance and colorectal cancer. World J Gastroenterol, 22(2), 501-518. doi:10.3748/wjg.v22.i2.501

31. Rubinstein, M. R., Wang, X., Liu, W., Hao, Y., Cai, G., \& Han, Y. W. (2013). Fusobacterium nucleatum promotes colorectal carcinogenesis by modulating $\mathrm{E}$-cadherin/ $\beta$-catenin signaling via its FadA adhesin. Cell Host Microbe, 14(2), 195-206. doi:10.1016/j. chom.2013.07.012

32. Coppenhagen-Glazer, S., Sol, A., Abed, J., Naor, R., Zhang, X., Han, Y. W., \& Bachrach, G. (2015). Fap2 of Fusobacterium nucleatum is a galactose-inhibitable adhesin involved in coaggregation, cell adhesion, and preterm birth. Infect Immun, 83(3), 1104-1113. doi:10.1128/IAI.02838-14

33. Abed, J., Emgård, J. E., Zamir, G., Faroja, M., Almogy, G., Grenov, A., .. . Bachrach, G. (2016). Fap2 Mediates Fusobacterium nucleatum Colorectal Adenocarcinoma Enrichment by Binding to Tumor-Expressed Gal-GalNAc. Cell Host Microbe, 20(2), 215-225. doi:10.1016/j.chom.2016.07.006

34. Jin, C., Lagoudas, G. K., Zhao, C., Bullman, S., Bhutkar, A., Hu, B., . . Jacks, T. (2019). Commensal Microbiota Promote Lung Cancer Development via үठ் T Cells. Cell, 176(5), 998-1013.e1016. doi:10.1016/j.cell.2018.12.040

35. Arismendi Sosa, A. C., Salinas Ibáñez, A. G., Pérez Chaca, M. V., Penissi, A. B., Gómez, N. N., \& Vega, A. E. (2018). Study of Helicobacter pylori infection on lung using an animal model. Microb Pathog, 123, 410-418. doi:10.1016/j.micpath.2018.07.038

36. Gui, Q. F., Lu, H. F., Zhang, C. X., Xu, Z. R., \& Yang, Y. H. (2015). Well-balanced commensal microbiota contributes to anticancer response in a lung cancer mouse model. Genet Mol Res, 14(2), 5642-5651. doi:10.4238/2015.May.25.16

37. Lakritz, J. R., Poutahidis, T., Mirabal, S., Varian, B. J., Levkovich, T., Ibrahim, Y. M., . . Erdman, S. E. (2015). Gut bacteria require neutrophils to promote mammary tumorigenesis. Oncotarget, 6(11), 9387-9396. doi:10.18632/oncotarget.3328

38. Zhu, J., Liao, M., Yao, Z., Liang, W., Li, Q., Liu, J., . . Mo, Z. (2018). Breast cancer in postmenopausal women is associated with an altered gut metagenome. Microbiome, 6(1), 136. doi:10.1186/ s40168-018-0515-3

39. Mabrok, H. B., Klopfleisch, R., Ghanem, K. Z., Clavel, T., Blaut, M., \& Loh, G. (2012). Lignan transformation by gut bacteria lowers tumor burden in a gnotobiotic rat model of breast cancer. Carcinogenesis, 33(1), 203-208. doi:10.1093/carcin/bgr256

40. Sakamoto, K., Schmidt, J. W., \& Wagner, K. U. (2015). Mouse models of breast cancer. Methods Mol Biol, 1267, 47-71. doi:10.1007/978-1-4939-2297-0_3

41. Humphries, A., \& Daud, A. (2018). The gut microbiota and immune checkpoint inhibitors. Hum Vaccin Immunother, 14(9),
2178-2182. doi:10.1080/21645515.2018.1442970

42. Oancea, I., Movva, R., Das, I., Aguirre de Cárcer, D., Schreiber, V., Yang, Y., . . . Florin, T. H. (2017). Colonic microbiota can promote rapid local improvement of murine colitis by thioguanine independently of T lymphocytes and host metabolism. Gut, 66(1), 59-69. doi:10.1136/gutjnl-2015-310874

43. Routy, B., Le Chatelier, E., Derosa, L., Duong, C. P. M., Alou, M. T., Daillère, R., . . Zitvogel, L. (2018). Gut microbiome influences efficacy of PD-1-based immunotherapy against epithelial tumors. Science, 359(6371), 91-97. doi:10.1126/science.aan3706

44. Yi, M., Yu, S., Qin, S., Liu, Q., Xu, H., Zhao, W., . . Wu, K. (2018). Gut microbiome modulates efficacy of immune checkpoint inhibitors. J Hematol Oncol, 11(1), 47. doi:10.1186/s13045-0180592-6

45. Arrieta, M. C., Stiemsma, L. T., Dimitriu, P. A., Thorson, L., Russell, S., Yurist-Doutsch, S., . . Investigators, C. S. (2015a). Early infancy microbial and metabolic alterations affect risk of childhood asthma. Sci Transl Med, 7(307), 307ra152. doi:10.1126/ scitransImed.aab2271 